\title{
Forum on frontiers of science and technology: Lifeomics and translational medicine
}

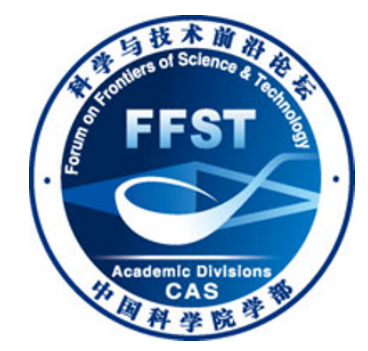

\author{
QIANG BoQin ${ }^{1}, \mathrm{HE} \mathrm{FuChu}^{2^{*}} \&$ ZENG YiXin ${ }^{3}$ \\ ${ }^{1}$ State Key Laboratory of Medical Molecular Biology, Institute of Basic Medical Sciences, Chinese Academy of Medical Sciences, \\ Beijing 100005, China; \\ ${ }^{2}$ State Key Laboratory of Proteomics, Beijing Proteome Research Center, National Center for Protein Sciences (Beijing), \\ Beijing 100850, China; \\ ${ }^{3}$ State Key Laboratory of Medical Molecular Biology, Chinese Academy Of Medical Sciences, Peking Union Medical College, \\ Beijing 100730, China
}

Received February 20, 2013

Citation: $\quad$ Qiang B Q, He F C, Zeng Y X. Forum on frontiers of science and technology: Lifeomics and translational medicine. Sci China Life Sci, 2013, 56: 199-200, doi: 10.1007/s11427-013-4463-7

The Chinese Academy of Sciences held its 7 th forum on frontiers of Science and Technology, April 12-13, 2012, in the academy hall of the Chinese Academy of Sciences. The Forum was organized by the Life Science and Medicine Division and co-organized by Academy of Military Medical Sciences and Science China Press. The theme of the 7th Forum was "Lifeomics and Translational Medicine", and the purpose was to discuss the significance of lifeomics (i.e., genomics, RNAomics and proteomics) in life sciences, to examine the current status, and identify the trends of lifeomics, to promote inter-disciplinary collaboration and transformation, and to stimulate international academic exchanges and cooperation. An unprecedented number of 12 academicians participated in the forum. They were Academicians Qiang BoQin, He FuChu, and Zeng YiXin, Shi YunYu, Chen KaiXian, Zhao GuoPing, He Lin, Chen RunSheng, Chen XiangMei, Yang HuanMing, Cheng Jing, and Zhang XueMin. Academicians Qiang BoQin, He FuChu, and Zeng YiXin served as co-chairs, Other participants included Profs. Wu JiaRui, Zhu WeiMin, Wang Jun, Han JingDong, Chen Biao, Han ZeGuang, He DaCheng, Jiang Ying, Li Dong, Liang SongPing, Liu ShaoJun, Liu SiQi, Liu YinKun, Luo JingChu, Peng XiaoZhong, Qian

*Corresponding author (email: hefc@bmi.ac.cn)
XiaoHong, Qin Jun, Qu LiangHu, Sun Wei, Wang Jian, Wei KaiHua, Xu Ping, Yang XiaoMing, Zhao ShiMin, Zhou GangQiao, Zhu JingDe, and Zhu YunPing, all of whom are leaders in this field. The forum attracted more than 200 expert attendees.

At the start of the forum, 15 well-known experts in lifeomics and translational medicine delivered excellent seminars focusing on "the rapid development of lifeomics", "lifeomics and bioinformatics" and "lifeomics and translational medicine". They reviewed the current status of genomics, RNAomics and proteomics. The Forum fostered discussions on advancing the application of the prospering lifeomics and translational medicine to help better understand the biology behind human diseases. At the second stage of the forum, discussions, dubbed "brainstorming", focused on the status quo and the developmental trends of lifeomics in the future, bioinformatics, as well as the combination of lifeomics and translational medicine.

After the brainstorming discussion, experts reached consensus on the following: (i) lifeomics (genomics, RNAomics and proteomics) would still be a new scientific discipline, employing unique epistemology and methodology, and quickly becoming the thrust of life sciences. Lifeomics would bring new perspective for life sciences and medical research and would be therefore, of significant value with 
an excellent prospect in application; (ii) lifeomics technologies based on high-throughput gene sequencing and biological mass spectrometry were set to open up new avenues of research and to usher in a new era of discovery in life sciences. The ever-advancing lifeomics technologies would certainly speed up the output rates, quality and accuracy of large-scale quantitative omics data, providing us with oceans of data, but would also bring forward some new issues, such as individual differences and personalized medicine, environmental factors and epigenetics and diseases, omics and evolution; (iii) standardization of the omics information and capabilities of in-depth data mining would become current technological bottlenecks. What was urgently needed was to address those issues by both technological and scientific mechanisms; (iv) lifeomics and translational medicine had forged a preliminary unification, started exploration in mechanisms, diagnosis and treatment of complex diseases such as cancer, diabetes, and hepatitis.

Participating experts also presented some of their own views about lofeomics and suggested ways to manage the research in omics. As for how to develop lifeomics strategically, the following were recommended: (i) the main tasks of lifeomics would be to describe the phenomenon of life, to investigate the physiological mechanisms, and to apply the omics in medicine and human health. Three stages of lifeomics development were envisioned, which were technology development, functional studies, and translational medicine; (ii) the study of lifeomics in China was generally fragmented, lacking cohesion. It would be of great importance that research efforts be coordinated to facilitate effective communication and integration amongst research institutions and between research institutions and hospitals; (iii) we should engage in lifeomics research with Chinese characteristics, for example, omics in traditional Chinese medicine, and systems biology of stem cell differentiation induciton.

As to the scientific prospects of lifeomics, some participants believed that we should focus on (i) the impact of various types of modification on the function of biomolecules (i.e. DNA, RNA, and protein); (ii) omics research on cancer and other complex diseases, including the relationship between the tumor and the evolution, cancer and aging, and new cancer treatment targets; (iii) the interaction omics, including expanded protein-protein interaction networks, the interaction of protein with other molecules, signaling network function and regulation, and network pharmacology; (iv) comprehensive study of lifeomics with an aim to integrate genomics and RNA-omics with proteomics, glycomics and metabolomics; (v) preliminary exploration on personalized treatment with minimizing the costs in mind.

Experts at the Forum also believed that our research efforts should address the following: (i) establishing bioinformatics data standards and data management centers; In omics research, China was not lagging behind but leading the world in some aspects. However, in data standards and management, there was a large gap between China and the developed countries in Europe and the United States. The quantity and quality of the data fell short of the research needs. Besides, the variety of quality standard format and the lack of sharing mechanisms further hindered our progress. Priority must be given to developing data standards, data submission and sharing mechanisms, and to building bioinformatics service platforms for multi-omics research; (ii) omics service platforms. Omics in China was still at the stage where each research team still had the mindset of doing everything themselves, which to some extent affected the quality control and public sharing of data. The urgent need would be to make full use of excellent teams in order to strengthen the open services and to promote the optimal allocation and efficient use of scientific resources; (iii) human cohort. Human cohort would not only be an important resource for functional omics research but could also serve as a bridge between bench and bed; (iv) omics of model organisms, especially primates. Studies of model organisms would prepare us for human omics research.

The large-scale nature of omics research brings in management issues including how to finance the projects. The recommendations are as follows: (i) we should take advantage of our tradition in big projects to initiate a major omics project with academician He FuChu serving as the chief scientist. Research efforts of national teams with excellence in genomics, RNAomics and proteomics would be coordinated to focus on pressing scientific issues; (ii) an coordinated thinking and planning would be necessary to reflect the ultimate goal of lifeomics and current technical status. Top-down design of research policies and programs were in need and implementing research programs in stages was recommended; (iii) centering around major diseases, large research teams should be established integrating research institutions and hospitals; (iv) innovation in the mechanisms assessing research institutions, universities and colleges, and hospitals would be necessary in order to promote translational medicine; (v) innovation in management mechanisms for omics service centers, especially data centers, would be a must in order to have better and open service, to have better quality control over data collection, and sharing.

Open Access This article is distributed under the terms of the Creative Commons Attribution License which permits any use, distribution, and reproduction in any medium, provided the original author(s) and source are credited. 